OPEN ACCESS

Edited by:

Dongmei Zhang,

Jinan University, China

Reviewed by:

Hao Zhang,

University of Pennsylvania,

United States

Lingzhi Li,

University of Texas MD Anderson

Cancer Center, United States

*Correspondence:

Leila Jafari

l.jafarie67@gmail.com

Zhe-Sheng Chen

chenz@stjohns.edu

tThese authors have contributed equally to this work

Specialty section:

This article was submitted to

Cellular Biochemistry,

a section of the journal

Frontiers in Cell and Developmental

Biology

Received: 29 August 2021

Accepted: 16 September 2021

Published: 06 October 2021

Citation:

Izadirad M, Huang Z, Jafari F, Hamidieh AA, Gharehbaghian A, Li Y-D, Jafari $L$ and Chen Z-S (2021)

Extracellular Vesicles in Acute Leukemia: A Mesmerizing Journey

With a Focus on Transferred

microRNAs.

Front. Cell Dev. Biol. 9:766371. doi: 10.3389/fcell.2021.766371

\section{Extracellular Vesicles in Acute Leukemia: A Mesmerizing Journey With a Focus on Transferred microRNAs}

\author{
Mehrdad Izadirad ${ }^{1+}$, Zoufang Huang ${ }^{2 \dagger}$, Farideh Jafari', Amir Ali Hamidieh ${ }^{3}$, \\ Ahmad Gharehbaghian', Yi-Dong Li ${ }^{4}$, Leila Jafari ${ }^{3 *}$ and Zhe-Sheng Chen ${ }^{4,5 *}$ \\ ${ }^{1}$ Department of Hematology and Blood Bank, School of Allied Medical Sciences, Shahid Beheshti University of Medical \\ Sciences, Tehran, Iran, ${ }^{2}$ Department of Hematology, The First Affiliated Hospital of Gannan Medical University, Ganzhou, \\ China, ${ }^{3}$ Pediatric Cell and Gene Therapy Research Center, Gene, Cell and Tissue Research Institute, Tehran University \\ of Medical Sciences, Tehran, Iran, ${ }^{4}$ Department of Pharmaceutical Sciences, College of Pharmacy and Health Sciences, \\ Queens, NY, United States, ${ }^{5}$ Institute for Biotechnology, St. John's University, Queens, NY, United States
}

Despite their small size, the membrane-bound particles named extracellular vesicles (EVs) seem to play an enormous role in the pathogenesis of acute leukemia. From oncogenic hematopoietic stem cells (HSCs) to become leukemic cells to alter the architecture of bone marrow (BM) microenvironment, EVs are critical components of leukemia development. As a carrier of essential molecules, especially a group of small non-coding RNAs known as miRNA, recently, EVs have attracted tremendous attention as a prognostic factor. Given the importance of miRNAs in the early stages of leukemogenesis and also their critical parts in the development of drug-resistant phenotype, it seems that the importance of EVs in the development of leukemia is more than what is expected. To be familiar with the clinical value of leukemia-derived $\mathrm{EVs}$, this review aimed to briefly shed light on the biology of EVs and to discuss the role of EV-derived miRNAs in the development of acute myeloid leukemia and acute lymphoblastic leukemia. By elaborating the advances and challenges concerning the isolation of EVs, we discuss whether EVs could have a prognostic value in the clinical setting for leukemia.

Keywords: acute myeloblastic leukemia, acute lymphoblastic leukemia, extracellular vesicles, prognosis factor, disease pathogenesis, miRNAs, non-coding RNAs

\section{INTRODUCTION}

As the most common type of leukemia, the incidence of acute leukemia, either acute myeloid leukemia (AML) or acute lymphoblastic leukemia (ALL), is increased in the last few years (Hunger and Mullighan, 2015; Park et al., 2015; Terwilliger and Abdul-Hay, 2017; Bosshard et al., 2018; Wiese and Daver, 2018). The aggressive and heterogeneous behavior of acute leukemia is originated from not only the diversity of genetic abnormalities but also the occurrence of extensive epigenetic changes. The list of involved genes and molecules in the pathogenesis of these malignancies is endless and new candidates are continuously emerging. This heterogenic characteristic of acute 
leukemia brings obstacles in the way of precise treatment of the disease and somehow ends the era of the conventional chemotherapeutic approaches (Bassan and Hoelzer, 2011; Selim and Moore, 2018). Despite the efficacy of the common chemotherapy regimen consist of cytarabine and anthracycline, which thus far is the most effective treatment strategy for leukemia patients, a considerable proportion of patients succumb to the disease due to the therapy failure (Bassan and Hoelzer, 2011; Luger, 2017).

Recently, a group of extracellular vesicles (EVs) derived from the cancer cells that carry the property of their parental cells ranging from signaling proteins to nucleic acids such as DNAs was successfully identified (Voso et al., 2019). It became evident that cancer cell-derived EVs are auxiliary tools that support cancer growth by carrying the factors that could enhance angiogenesis (Skog et al., 2008), provide the metabolic needs of tumor cells and promote their proliferative capacity (Fong et al., 2015). In hematologic malignancies, for example in leukemia, EVs were showed to participate in the primary tumor growth as well as inducing multi-drug resistance through recruiting microenvironment resident cells such as endothelial cells (ECs) or leukocytes (Litwińska et al., 2019). In ALL, it has been proposed that the communication between leukemic cells and the bone marrow residential cells could increase the survival of leukemic cells through exporting exo-RNAs. In pre-B ALL, for example, some evidence revealed that the delivered exo-miRNAs could change the architecture of the BM environment in the manner that it reinforces the proliferation of leukemic cells and suppresses the activity of immune system (Xu et al., 2018). MicroRNAs are one of the main non-coding RNAs that play a critical role in the development of leukemia. There are multiple evidence reporting that miRNA transferred by EVs into the leukemic cells could be fundamental in the pathogenesis of leukemia. For example, the transferred miR155 to AML cells could suppress the expression of C/EBPA to reinforce the development of leukemogenesis (Alemdehy et al., 2016). Or, the upregulation of long non-coding RNA SNHG1 expression has also been suggested to elevate the growth of the AML cells through targeting miR-489-3p (Li et al., 2021). The transfer of miR-181 family to ALL cells has also been suggested to be associated with CNS involvement (Egyed et al., 2020). Moreover, it should be noted that not all the transfer of miRNAs are participated in the early stages of leukemogenesis and sometimes the transferred miRNAs are responsible of protecting the leukemic cells from the devastating effects of the anti-cancer agents. In this vein, it became evident that exosomes carrying miR-155 could confer drug-resistance against tyrosine kinase inhibitors in AML cells (Viola et al., 2016). The transfer of miR-19b and miR-20a has also been reported to be associated with induction of drug-resistance in APL cells through increasing the expression of multiple drug resistant proteins (MDPs) (Bouvy et al., 2017).

In addition to their key roles in the pathogenesis of acute leukemia, EVs are also a valid repertoire of genetic and epigenetic abnormalities of the parental cells. The next-generation sequencing (NGS) and GeneScan-based fragment-length analysis on the identified double-stranded DNA (dsDNA) in
AML-derived EVs revealed that the majority of dsDNA mirror the mutations found in the genomic DNA obtained from primary leukemia cells (Kontopoulou et al., 2020; Bernardi et al., 2021). It has been suggested that there is a similar pattern between the expression of miRNAs in the EVs and pre-B ALL-derived leukemic cells (Xu et al., 2016). These findings together with the fact that these membrane bilayer-enclosed structures could be reachable in the body fluids suggest EVs as a valid tool for early detection, and for predicting patient outcome of both AML and ALL. The disease diagnosis/prognosis is not the only area that leukemia-derived EVs could be employed, as these groups of microvesicles could also integrate into the risk stratification of patients and even in the therapeutic strategies. To understand the clinical value of leukemia-derived EVs, this review aims to summarize the biology of EVs and discuss how EV-derived miRNAs could play a part in the development of AML and ALL. By combining the advances and challenges concerning the isolation of EVs, we discuss whether EVs could have a prognostic value in the clinical setting for leukemia patients.

\section{THE BIOLOGY AND FUNCTION OF EXTRACELLULAR VESICLES}

\section{The Origin of Extracellular Vesicles}

The evidence of the existence of EVs dated back to almost 60 years ago when the 20-50 nm-sized vesicles carrying clotting factors were identified in human platelet-free plasma (Wolf, 1967). In an article published by Wolf (1967), these lipid-rich vesicles were described as minute particulate material referred to as platelet dust because they were released from platelet (PLTs) during activation. The results of electronic microscopy revealed that these particles carry phospholipids and platelet factor 3 (tissue factor) to facilitate the coagulation process (Wolf, 1967). Since then, the definition and the perspective of this particle which is now named as EVs has changed. Now, EVs are envisioned as a group of lipid bilayer-enclosed structures that are released into the extracellular space (Doyle and Wang, 2019; Cocozza et al., 2020; Kalluri and LeBleu, 2020) by most cells to either discard their unwanted products or communicate with other neighboring or distant cells and even with extracellular matrix (ECM) (Doyle and Wang, 2019; Cocozza et al., 2020). This means that the content of EVs could be varied from proteins, lipids, and nucleic acids that could change the behavior of the recipient cells (Doyle and Wang, 2019; Cocozza et al., 2020). Thus far, several types of EVs have been identified in biological fluids such as urine, blood, ascites, and cerebrospinal fluid (Ibrahim and Marbán, 2016), and according to their size, these vesicles are classified into three groups: exosomes with the size range from 30 to $150 \mathrm{~nm}$, apoptotic bodies that have the size of 50-5,000 nm, and microvesicles (also known as ectosomes, shedding vesicles, or microparticles) with $100-1,000 \mathrm{~nm}$ in diameter (Kim et al., 2018; Liu et al., 2021). Apart from the size, the mechanism of release of $\mathrm{EV}$ differs from each other. While exosomes require multivesicular bodies (MVBs) for transportation, apoptotic bodies and microvesicles are released through interacting with the plasma membrane (Hessvik and Llorente, 2018). Among 
the mentioned EVs, it should be noted that exosomes have the most participation in intercellular communication, neuronal communication, antigen presentation, immune responses, organ development, and reproductive performance. This means that exosomes may have the most fundamental roles in pathophysiologic conditions, as well (Crenshaw et al., 2018; He et al., 2018). However, it should be noted that the size classification could not properly distinct microvesicles from exosomes, as not only both vesicles may overlap in the size but also both could be found in extracellular fluids and may exert similar intracellular effects (Zaborowski et al., 2015). Given these, in most cases, both microvesicles and exosomes are referred to as circulating vesicles (Raposo and Stoorvogel, 2013).

\section{Biogenesis of Circulating Vesicles}

Like their intracellular functions, the biogenesis of circulating vesicles is complex and numerous internal and external factors are involved in this process. Different membrane receptors, lipid raft complexes, and endosomal sorting complexes regulate the biogenesis of EVs; however, this process could not be accomplished without the presence of Ras superfamily GTPase (Kim et al., 2018; Jadli et al., 2020).

\section{Biogenesis of Exosomes}

The biogenesis of exosomes includes three main steps; early endosomes (EEs) and late endosomes (LEs) or MVB formation, intraluminal vesicles (ILVs) formation, and the secretion of ILV into the extracellular space.

Early endosomes (EEs) form as a result of membrane budding inward the cell. The generated EEs subsequently fuse with endocytic vesicles to incorporate their cargos. After the evacuation of recycling endosomes from EEs, they undergo several stages of maturation to produce late endosomes (LEs), also known as multivesicular bodies (MVBs). There are two destinies for MVBs; either merge with the lysosome which leads to their destruction, or package their contents as $30-100 \mathrm{~nm}$ vesicles named intraluminal vesicles (ILVs) to integrate with the plasma membrane and release into the extracellular space (Akers et al., 2013). In this process, the companionship of several factors including cytoskeleton, motors proteins, and Rab family of GTPases may decide whether MVBs move toward lysosome or plasma membrane. For example, while ubiquitylated 7 guides MVBs toward lysosomal degradation, the members of the Rab family of GTPases such as Rab27A, Rab11, and Rab35 regulate the fusion of MVBs into the membrane through generating ILVs (Ostrowski et al., 2010).

The formation of ILVs consists of two main steps. At the primary stage, the endosome membrane should be organized by specific transmembrane proteins known as Tetraspanins (Pols and Klumperman, 2009). The presence of Tetraspanins is critical for the formation of ILVs, as these proteins construct Tetraspanin enriched microdomains (TEMs) domains within the membrane which could later cluster the essential proteins for ILVs generation (Hemler, 2005). Thus far, CD9 and CD63 are the most common as well as important Tetraspanins on the surface of ILVs that are also used as an identifier to isolate EVs in the body fluids (Jansen et al., 2009; Kosaka et al., 2010). The second step of ILVs formation is allocated to the addition of a group of multi-protein complexes named endosomal sorting complex required for transport (ESCRTs). The results of the in-depth molecular investigations showed that the presence of 4 types of ESCRT called ESCRT 0, I, II, and III is essential for membrane budding. Among them, the presence of ESCRT III is necessary for complete membrane budding. Upon EE formation and cargo mono-ubiquitination (Crenshaw et al., 2018; Mashouri et al., 2019), ESCRT-0 in the complex with hepatocyte growth factor-regulated tyrosine kinase substrate (HRS) and STAM1/2 recruit ESCRT I/II to bind to the phosphatidylinositol 3-phosphate (PIP3) located on the membrane. This event curves the membrane inward the lumen and also recruits the complex of ESCRT III-Alix- TSG101 that is essential for complete secretion of the vesicle from the membrane (Crenshaw et al., 2018; Juan and Furthauer, 2018; Mashouri et al., 2019; Jadli et al., 2020). It should be noted that if the cargo does not undergo monoubiquitination, ALG-2 interacting protein-X (ALIX) forms a team with Syntenin-Syndecan complex (Juan and Furthauer, 2018) or PAR1 (Kim et al., 2018; Skryabin et al., 2020) to mediate ESCRT-dependent secretion of exosomes (Kim et al., 2018; Mashouri et al., 2019).

Previous studies showed that even in the absence of HRS, Alix, and TSG101, the biogenesis of exosomes is continued. It has been suggested that this process could be mediated independently of ESCRT complex and through raft-dependent mechanisms. Sphingomyelin is a key player in this process (Skryabin et al., 2020), as its cleavage via sphingomyelinase 2 produces ceramide, a waxy lipid molecule which facilitates the formation of ILVs through regulating membrane budding (Juan and Furthauer, 2018). Moreover, ceramide could be catalyzed into sphingosine 1-phosphate (S1P) (Kajimoto et al., 2013), a signaling molecule which attracts cargos such as CD63, CD81, and flotillin into ILVs via interaction with inhibitory $\mathrm{G}$ protein $(\mathrm{Gi})$-coupled receptor. No matter through which mechanism ILVs may be produced within the MVBs. NSF attachment protein receptors (SNAREs) provide a platform for their secretion into the extracellular space. Through binding of $\mathrm{Ca}^{2+}$ to synaptotagmin VII, SNAREs complex could be activated, resulted in secretion of EVs (van Niel et al., 2018; Jadli et al., 2020). When the exosomes were released by the parental cells, VPS4 separates the remained components to recycle them for further uses.

\section{Biogenesis of Micro Vesicles and Apoptotic Bodies}

Since micro vesicles (MV) differ from the size from the exosomes, their biogenesis might have some differences with EVs. In the biogenesis of $\mathrm{MV}$, actin rearrangement play a fundamental role (Gurunathan et al., 2021). In GTPase-dependent pathway, an enzyme named LIM kinase (LIMK) adds a phosphoryl group cofilin, which is a to actin depolymerizing enzyme, and thereby by inactivating this enzyme facilitate MV budding ( $\mathrm{Li} \mathrm{B}$. et al., 2012). Another mechanism that could lead to MV biogenesis could be mediated through Small GTPase, ADP-ribosylation factor 6 (ARF6) signaling. Once ARF6 is activated, it could recruit ERK signaling pathway to activate myosin light-chain kinase (MLCK), which in turn activates myosin light-chain (MLC) at the necks of MVs. Activated MLCK interact with 
actin filaments, a process leads to MV production (MuralidharanChari et al., 2009). The biogenesis of MV could be triggered by phospholipid redistribution or cytoskeleton reorganization (Akers et al., 2013; D'Souza-Schorey and Schorey, 2018). The best example of a biological process that could activate MV biogenesis is the translocation of phosphatidylserine (PS) to the out membrane, a process that occur in the apoptosis (Akers et al., 2013; D'Souza-Schorey and Schorey, 2018). In apoptosis, cellular contents are packed in the form of small membrane-bound vesicles known as apoptotic bodies (ApoBDs), which contains externalized phosphatidylserine, calreticulin, and calnexin (Nunez et al., 2010). When actin-myosin located at the membrane starts to concentrate, a mechanism leads to membrane blebbing, the formation of ApoBDs begins (Xu et al., 2019). In addition to membrane blebbing, the reduction in the volume-tosurface ratio of cells also is another factor that provoke ApoBDs formation (Nunez et al., 2010).

\section{Extracellular Vesicles as a Vehicle to Deliver Essential Components to Target Cells}

Based on the type of the parental cells and the mission that EV may have, the cargo of these circulating vesicles varies. However, proteins, lipids, metabolites, RNAs, and cDNAs are the common components of EVs (Crenshaw et al., 2018; He et al., 2018; Huang and Deng, 2019). Table 1 listed the most common molecules that could be detected in EVs, irrespective of their origin and cellular function.

\section{Proteins}

Apart from structural proteins such as tetraspanins (CD81, CD82, CD37, and CD63), TSG101, Alix, and Rab family, heat shock protein, clathrin, protein kinase G (PKG), ATPase, syntenin, and RNA binding proteins (RBPs) are the most common types of proteins that are detected in EVs (Crenshaw et al., 2018; Huang and Deng, 2019; Jeppesen et al., 2019). It should be noted that the protein content of EVs depends on the original cell ( $\mathrm{He}$ et al., 2018). MHC class II, ICAM-1, integrin, CD20, PD-L1, EGFR, IGF-1R, and cytokine receptors are among the most important proteins that are isolated from EVs (Pegtel and Gould, 2019).

\section{Nucleic Acids}

One of the most important contents of EVs is nucleic acids that could be either mitochondrial DNA (mtDNA), double strands DNA (dsDNA), single strands DNA (ssDNA) (Kalluri and LeBleu, 2020), or different types of RNAs ranging from coding RNAs such as mRNAs to non-coding species (Chu et al., 2020; Kalluri and LeBleu, 2020; Skryabin et al., 2020). Among different types of nucleic acids, it seems that microRNAs (miRNAs) are the most important cargos of EVs. Carolina Villarroya-Beltri and colleagues have successfully reveal that sumoylated hnRNPA2B1 and Ceramide are critical for loading miRNAs into EVs (Kosaka et al., 2010; Villarroya-Beltri et al., 2013). Upon integrating with mRNAs in the recipient cells, miRNAs could conveniently change their cellular behaviors. This process is well-defined in cancer cells, where the exosome miRNAs provide a platform for cancer cells to grow, invade into distant organs, and resist chemotherapeutic drugs (Ingenito et al., 2019; Rahbarghazi et al., 2019). Recently, it has been indicated that the resident-long noncoding RNAs in EVs could epigenetically alter the behavior of target cells (Wang et al., 2019; Da et al., 2021). The list of the most common miRNAs and lncRNA in EVs can be found in Table 1.

\section{MicroRNAs}

miRNAs are the best tools for regulating gene expression, either transcriptionally or post-transcriptionally (Huntzinger and Izaurralde, 2011; O'Brien et al., 2018). In complex with other proteins, which is called as MiRISC [miRNA and Argonaute 2 (AGO-2)], the $5^{\prime}$-proximal region (nucleotide 2-8) of the miRNA binds to the $3^{\prime}$ UTR of the targeted mRNA and thereby inhibit or stimulate their expression. It should be noted that not all miRNAs bind to $3^{\prime}$ UTR of the mRNA and in some cases, miRNAs such as miR-10a could bind to $5^{\prime}$ UTR of the targeted mRNA (Ørom et al., 2008; Valinezhad Orang et al., 2014). Once miRNA interact to its targeted mRNA and if miRNA and miRNA response elements (MRE) are entirely complementary,

TABLE 1 | The content of circulating EVs.

Types of cargo

Common contents

\section{Proteins}

Structural proteins

Outer membrane lipid-anchored proteins

Inner membrane lipid-anchored proteins

Cell signaling proteins

Enzymes

LncRNAs

miRNAs
Tetraspanin proteins (CD81, CD82, CD37, and CD63), Syntenin, Alix, tumor susceptibility gene 101 protein (TSG101), Syndecans (SDC1-4), Intercellular adhesion molecule-1 (ICAM-1), Integrins, and Chaperons. CD39, CD73, CD55, CD59, Glypican-cellular prion protein (PrPC), and Amyloidogenic conformer.

Small GTPases superfamily, and Protein kinases (Src).

Epidermal growth factor receptor (EGFR), Vascular endothelial growth factor receptor type-2 (VEGFR2), Insulin-like growth factor I receptor (IGF-1R), Notch receptors, Cytokine receptors, G protein-coupled receptors (GPCRs), Wnt proteins, Bone morphogenetic proteins (BMPs), Transforming growth factor $\beta$ (TGF- $\beta$ ), tumor necrosis factor (TNF), TNF-related apoptosis-inducing ligand, first apoptosis signal (FAS) ligand, and extracellular matrix (ECM) proteins.

Phosphatases, Pyrophosphatases, Calcium-binding annexins, and phosphate transporters, RNA editing enzymes, Lipases, Proteases, Glycosyltransferases, Glycosidases, and Metabolic enzymes.

lincRNA-p21, ANRASSF1, IncRNA SYSIL, IncRNA ROCK1, and IncRNA Paupar.

miR-150, -221, -1246, -140-3p, -16-5p, -20a-5p, -15a-5p, -17-5p, -18a, let-7b. 
the endonuclease activity of AGO-2 be provoked to cleave the mRNA (O'Brien et al., 2018). There are some piece of evidence suggesting that miRNAs could also enhance the expression of some genes (Huntzinger and Izaurralde, 2011). For example, when AGO makes a team with another protein associated to the miRNA-protein complex (microRNPs) named Fragile-x-mental, this complex could bind to AU-rich elements (AREs) at the $3^{\prime}$ UTR of the targeted mRNAs to enhance its expression. The best example of this regulatory process could be seen in Let-7 which by recruiting this mechanism could enhance the expression of the genes leading to cell cycle arrest (Forman et al., 2008). miR-24-1 is another miRNA that seems to could enhance the transcription of the target genes via inducing chromatin remodeling at enhancer site (Xiao et al., 2017). Given the importance of miRNAs in gene regulation, intense attention has been attracted to the biogenesis of these small non-coding RNAs.

\section{Biogenesis of MicroRNAs}

The biogenesis of miRNAs could be mediated through two main pathways; canonical and non-canonical mechanism (O'Brien et al., 2018). In canonical pathway, the primary miRNA (pri-miRNA) produced by RNA polymerase 2 converts to precursor-miRNA (pre-miRNA) by DROSHA/DiGeorge Syndrome Critical Region 8 (DGCR8) complex. The produced pre-miRNA, then, exported to the cytoplasm via exportin5, where it loses its pri-terminal miRNA's loop via RNase III endonuclease Dicer. Helicase, then, come to play to convert the mature double stranded miRNA to single stranded miRNA (Han et al., 2004; Okada et al., 2009). In non-canonical pathway; however, the biogenesis of miRNAs could be mediated through Drosha/DGCR8 and Dicer-independent manner (O'Brien et al., 2018). In this manner, the non-cleaved pre-miRNA recruit exportin 1 to transport into the cytoplasm, where it makes a team with Argonaute-2 (AGO-2) to become mature (O’Brien et al., 2018).

\section{Transfer of MicroRNAs to Extracellular Vesicles}

Through binding to either RNA-Binding Proteins (RBP) or membrane proteins, miRNAs could be packaged into EVs (Groot and Lee, 2020). For example, RNA-Binding Proteins (RBP) such as heterogeneous nuclear ribonucleoprotein A2B1 (hnRNPA2B1) binds miRNA to transfer them into the EVs (Villarroya-Beltri et al., 2013). AGO-2, apart from its role in miRNA maturation, not only could aid miRNAs to transfer into EVs by recruiting the KRAS-MEK-ERK signaling pathway (Li L. et al., 2012; McKenzie et al., 2016) but also could protect them from degradation (Groot and Lee, 2020). Synaptotagminbinding cytoplasmic RNA-interaction protein (SYNCRIP) is another protein that could join miRNA to transfer them into EVs. Through binding to extra-seed sequence (hEXO) motif of miRNAs, SYNCRIP aid miRNAs to gather into the exosomes (Santangelo et al., 2016).

\section{Extracellular Vesicles Transfer to Target Cells}

As a carrier of information to the recipient cells, EVs should be delivered properly as well as safely to the target cells. Indeed, the vesicular structure of EVs protects their contents from enzymatic degradation and guarantees that the cargo be delivered to the target cells in its original state. One striking point about EV secretion is that under a stressful condition, cells are more prone to produce EVs (King et al., 2012). This phenomenon could be explained in two ways; first, this is an attempt of the cells to discard the damaging and harmful factors, or second, this is a communicational tool to aware the neighboring cells of the ongoing event. Valid evidence exists for both of these hypotheses. It has been reported that in response to the DNA damage and as the result of $\mathrm{p} 53$ activation, the pace of EVs release is exacerbated (Yu et al., 2006). Also, during hypoxia, the tendency of the cells to produce EVs is much higher than normoxic conditions (Park et al., 2010). No matter what is the purpose of EV secretion, when these circulating vesicles are released, they should be delivered to the recipient cells. The membrane-bound activating or inhibitory molecules in EVs transmit a signal to the recipient cells to allow EVs entrance. Like other circulating vesicles, EVs also integrates with the membrane of the responder cells via either endocytosis or membrane fusion. Once EVs enter the cells, they evacuate their valuable biologically active molecules repertoire to alter the cellular behavior. For example, the delivered miRNAs or lncRNAs could epigenetically change the expression of a wide range of molecules, activate/suppress different signaling pathways, and change the chromosomal structure. Also, the transferred mRNA could be translated into proteins that previously did not exist in the responder cells (Parolini et al., 2009).

\section{EXTRACELLULAR VESICLES-DERIVED MICRORNAS, A TROJAN HORSE FOR CANCER DEVELOPMENT}

Although these seem to be striking methods to alter the characteristics of cells, production and delivery of EVs by cancer cells can not only transform the neighboring cells into the malignant counterpart but also engage the properties of the surrounding cells for their favor. The first evidence of the involvement of EVs in tumorigenesis was reported by Skog et al. (2008) who indicated that secreted EVs from glioblastoma cells enforce angiogenesis in brain endothelial cells. Very soon, the other pieces of evidence were found in other types of human cancers such as squamous cell carcinoma (Park et al., 2010), breast cancer (O’Brien et al., 2013; Zomer et al., 2015), and colorectal cancer (Tian et al., 2018), all suggesting that EVs act as Trojan horses to alter the microenvironment according to the needs of the tumor cells. Tumor-derived EVs could also induce immune exhaustion in the tumor microenvironment through upregulating the expression of inhibitory molecules of lymphocytes such as NK cells and CD8 positive T-lymphocytes or enhancing the differentiation of myeloid-derived suppressor cells (Lane et al., 2018). EVs also play a fundamental role in the pathogenesis of hematologic malignancies, as having a precise cross-talk with other residential cells within the BM niche is vital for the survival of hematologic malignant cells. The delivered EVs induce a "homing and nurturing" microenvironment in BM and protect the leukemic/neoplastic 

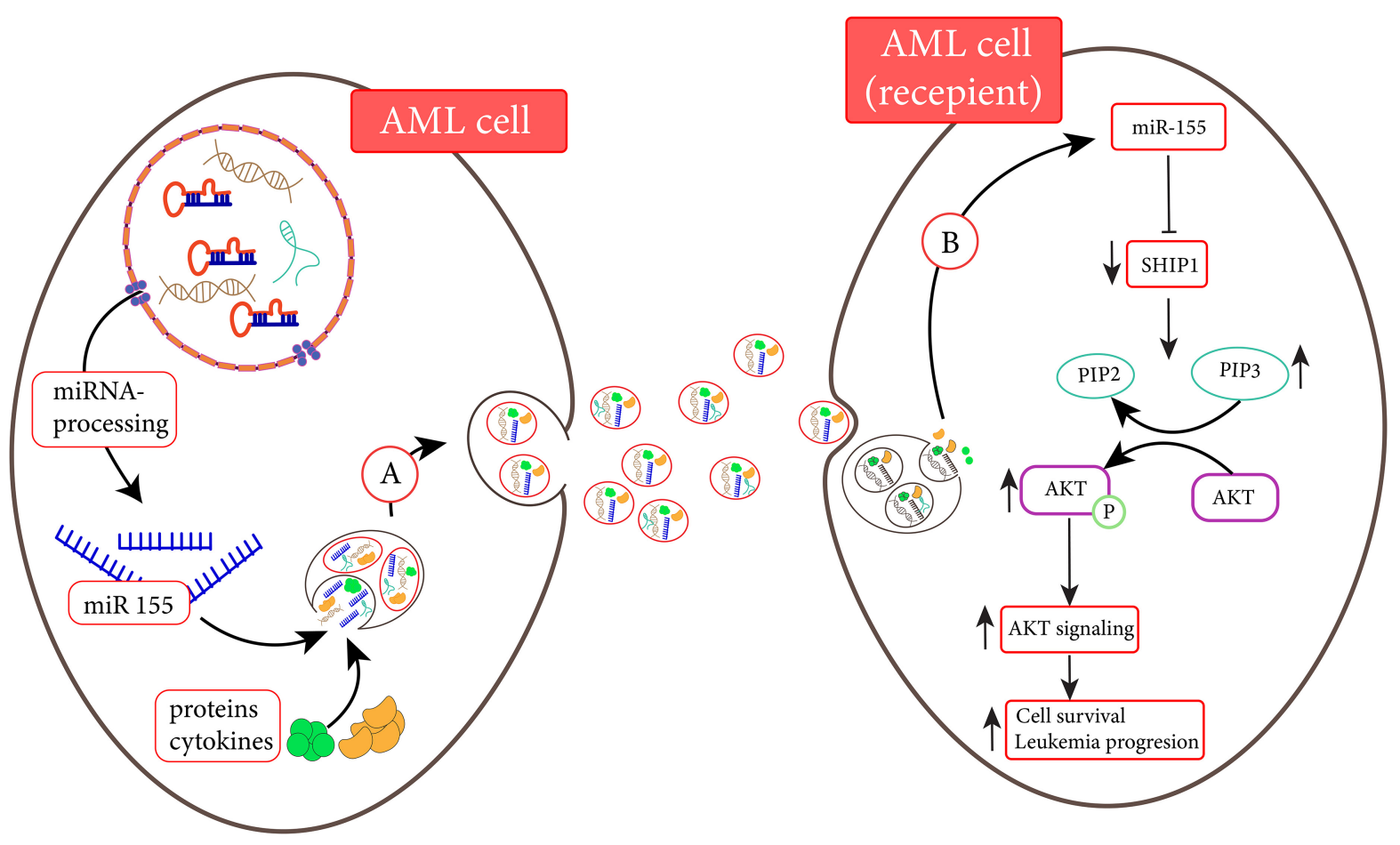

FIGURE 1 | AML cells are able to transfer miR-155 to another AML cell (Hornick et al., 2015). (A) MiR-155 and other elements are transferred to recipient cells via exosomes. (B) MiR-155 derived from exosomes is released into the recipient cell and inhibits the activated SH2 domain-containing inositol $5^{\prime}$-phosphatase1 (SHIP1) expression. Following the inhibition of SHIP1, the enhancement of AKT signaling promotes cell survival (Xue et al., 2014).

cells from the devastating effects of chemotherapeutic drugs. For example, it has been reported that chronic myeloid leukemia (CML)-derived EVs enforce BM stromal cells to produce IL-8, a cytokine which prolongs the survival of CML cells (Corrado et al., 2014). In multiple myeloma, it has been reported that the secreted EV from the BM mesenchymal stromal cells enhanced the proliferative capacity of multiple myeloma cells through transferring miR-15a (Roccaro et al., 2013). For acute type of leukemia (AML and ALL), there are multiple lines of evidence shedding light on the role of EVs in the pathogenesis of this common type of hematologic malignancy. In the following part of this article, we discuss the roles of EVs in the pathogenesis of acute leukemia.

\section{EXTRACELLULAR VESICLES AS A COMMUNICATION TOOL IN ACUTE LEUKEMIA}

\section{Extracellular Vesicles Participate in the Pathogenesis of Acute Myeloid Leukemia and Acute Lymphoblastic Leukemia}

In acute leukemia, EVs serve as a bridge to provide a dynamic cross-talk between leukemic cells and the stromal cells that reside in the BM niche. In another word, EVs are responsible for turning
BM microenvironment into a leukemia-permissive space. There are multiple evidence suggest that EVs might have a key role in the early stages of leukemogenesis. It has been revealed that the transfer of EVs from the leukemic cells to either HSCs or myeloid progenitor cells could abrogate the proper differentiation and thereby lead to the development of leukemia. Moreover, the transferred EVs protect leukemic cells from apoptotic stimuli such as chemotherapeutic drugs (Kumar et al., 2018). In the following part of the paper, more details will be discussed about the participation of EVs in the pathogenesis of leukemia.

\section{The Role of Extracellular Vesicles Derived MicroRNAs in the Regulation of Leukemogenesis}

One of the main mechanisms through which EVs promote the progression of leukemia is mediated through delivering the essential oncogenic RNAs into HSCs to change its characterization for developing into leukemic cells. In this process, the delivery of miRNAs plays fundamental roles. In ALL, for instance, it has been claimed that the leukemic cells released EVs containing miR-43a-5p to the BM microenvironment. After internalizing to the BMSCs, this miRNA targets Wnt signaling axis and thereby inhibits osteogenesis in the BM. The malignant HSCs transform to leukemic cells as osteogenesis is suppressed (Yuan et al., 2021). In addition, it has been found that the secreted EVs from BMSCs consisting of miR-21 are delivered into HSCs and consequently enhance the development of B-ALL cells. On the other hand, the exo-miR-21 could interact with TGF- $\beta$ 
and shut down the anti-tumor immune responses in the BM microenvironment ( $\mathrm{Lv}$ et al., 2021). The same mechanism was also observed in AML. A previous study showed that the serum level of EVs carrying miR-10b is elevated in AML patients as compared to the healthy counterparts (Fang et al., 2020). miR-10b is notorious for its role in halting the granulocytic/monocytic differentiation in HSCs and enhancing the proliferative capacity of immature myeloid progenitors, leading to AML development (Bi et al., 2018). The large amount of EVs containing miR-10b in newly diagnosed AML patients suggested the significant roles of this delivering system in inducing AML (Fang et al., 2020). MiR-4532 is another delivered miRNA that could be transferred to HSCs through AML-derived EVs to suppress the expression of LDOC1. LDOC1 is an inhibitor of the STAT-3 signaling pathway and thereby its downregulation leads to STAT-3 activation. When AML-derived EVs deliver such cargo to the HSCs, they manipulate the proliferative capacity of these cells by stimulating the STAT-3 signaling pathway (Zhao et al., 2019). The results of the previous studies also indicated that some of the leukemicderived EVs could induce early leukemogenesis in myeloid progenitors through transferring miR-155 (Figure 1). Through binding to 3/UTR of c-Myb, miR-155 inhibits the expression of this differentiating transcription factor in myeloid cells and thereby induces differentiation arrest, a critical step in AML development (Hornick et al., 2016). Apart from miRNAs, some evidence suggest the exosomal transfer of oncogenic mRNAs such as those encoding NPM1 and FLT3-ITD to the myeloid progenitors from leukemic cells could also lead to leukemia development (Huan et al., 2013). By silencing the expression of hematopoiesis-related growth factors such as IGF-1, CXCL12, KIT ligand, and IL-7, AML-derived EVs could enforce neoplastic HSCs committed to the myeloid progenitor, enhancing the production of leukemic cells (Kumar et al., 2018). The transfer of the anti-apoptotic proteins such as MCL-1, BCL-2, and BCL-XL to the immature myeloid blasts could also guarantee their survival in the BM microenvironment (Wojtuszkiewicz et al., 2016). In ALL, it has been found that leukemia-derived EVs could induce metabolic switch in BMSCs. Johnson et al. (2016) have proposed that the leukemia-derived EV recipient stromal cells have minimal mitochondrial aspiration and use an aerobic glycolysis instead of oxidative phosphorylation, which in turn could provide the desired energy for ALL development in the $\mathrm{BM}$ microenvironment. It seems that during leukemogenesis, EVs act as a Trojan horse by delivering either miRNAs or oncomRNAs. These tiny vesicles could alter the characteristic of BMSCs in a way that they increase the possibility of AML or ALL development.

\section{The Role of Extracellular Vesicles Derived MicroRNA in Regulating Survival of Leukemic Cells}

As mentioned earlier, one of the main purposes of EVs delivering in acute leukemia is to evolve a leukemia-permissive space in BM, where leukemic cells could have an opportunity to survive, proliferate and grow. The number of studies that cover this mechanism in the progression of both AML and ALL is skyrocketed over the last decades and thus far, numerous molecules have been identified to be involved in this process.
EVs could potentiate the survival and proliferative potential of ALL. In a study conducted by Patel et al. (2016), it has been reported that when non-proliferating ALL cells were cultured with $\mathrm{ph}^{+}$ALL-derived exosomes, their proliferative capacity were vigorously reinforced, suggesting that the contents of these EVs might have proliferative factors. But what components could be involved in this process? Haque and Vaiselbuh (2020) came up with the answer when they successfully isolated miR-181 from the EVs in the serum of pediatric cases of ALL. By conducting further analysis, they proposed that the delivered miR-181, on one hand could enhance the expression of anti-apoptotic proteins such as MCL-1 and BCL-2 in leukemic cells, and on the other hand, could elevate the expression of proliferationrelated genes, including PCNA and Ki-67. Moreover, the authors also claimed that the up-regulation of miR-181 in EVs-derived from ALL patients suppressed the expression of pro-apoptotic genes. As a straightforward interpretation of these results, it was proposed that ALL cells might have longer survival and more potent proliferative capacity by uptaking these EVs (Haque and Vaiselbuh, 2020).

For AML, multiple studies declared the importance of EVs in increasing the survival of leukemic cells. Through delivering DKK1 to the BM stromal cells (BMSCs), for example, AMLderived EVs could halt the progression of hematopoiesis and osteoblast differentiation in the $\mathrm{BM}$ niche and promote the uncontrolled proliferation of leukemic cells (Kumar et al., 2018). BMP-2 is another cargo that could be transmitted between AML cells and the mesenchymal stem cells (MSCs) in the BM microenvironment to guarantee the survival of leukemic cells. The excessive amount of BMP-2 in leukemic cells transfer in the form of EVs to MSCs, where this transcription factor could reinforce osteogenic differentiation. As a result, through secretion of connective tissue growth factor (CTGF) from MSCs, AML cells could find a chance to grow (Battula et al., 2017). So far, many exosome-delivered miRNAs have been identified for endowing the AML cells the survival advantages. MiR-125b, for instance, is one of these delivered miRNAs that target the expression of pro-apoptotic proteins such as BAK and Bmf, and P53 in AML cells (Figure 2). This miRNA could not only halt the induction of apoptosis in AML cells but also induce cell proliferation through promoting cell cycle (Bousquet et al., 2010; Zhang et al., 2011; Vargas Romero et al., 2015). Ji et al. (2021) have also suggested that BMSC-derived EVs could enhance AML development through delivering miR-26a-5p to leukemic cells. By activating the Wnt/B-catenin signaling pathway, the delivered miR-26a-5p promotes AML cell proliferation, migration, and invasion (Ji et al., 2021). Not all the alterations should be delivered by exo-miRNAs and in some cases exo-lncRNAs might also have a role in the regulation of the BM microenvironment. Exo-circ0009910 has been claimed to block the expression of miR-5195-3p and thereby enhance the progression of cell cycle in AML cells through up-regulating the growth factor receptor-bound protein 10 (GRB10) (Wang et al., 2021). Another exo-lncRNA that has been detected in the sera of AML patients is Circ-0004136, which is a sponge for miR-570-3p, a tumor suppressor miRNA that reduced the expression of TSPAN3 in AML cells. When EVs containing Circ-0004136 is delivered into AML cells, not only the 


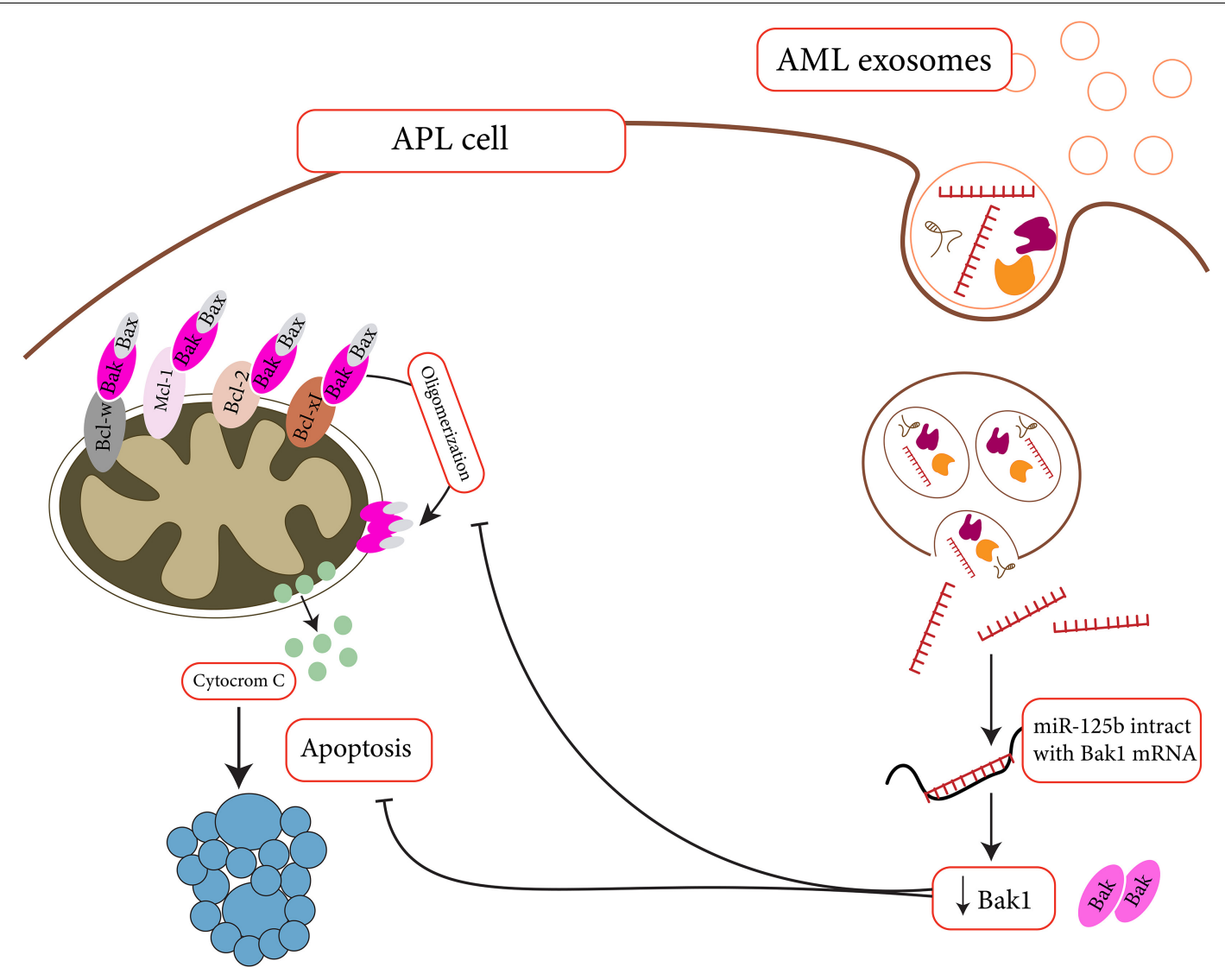

FIGURE 2 | Apoptosis suppression by miR-125b might help APL cells survive in cytotoxic situation (Zhang et al., 2011). MiR-125b is a microRNA that targets Bak1. As miR-125 levels rise, Bak levels fall, and apoptosis decreases.

viability of the cells be sustained but also these cells proliferate more autonomously (Bi et al., 2021).

\section{The Role of Extracellular Vesicles in Regulating Angiogenesis}

Since the density of BM microvessels is one of the main criteria that could protect the survival of leukemic cells in the $\mathrm{BM}$, it comes as no surprise that EVs might participate in the regulation of the density of $\mathrm{BM}$ microvesicles. Through delivering angiogenic factors/proteins and miRNAs, EVs change the characteristics of endothelial cells and promote angiogenesis (Huan et al., 2013). The angiogenic contents of EVs increase the proliferative capacity of endothelial cells, enforce their invasion and subsequently enhance the expression of proangiogenic factors such as IL6 and VEGF. Fang et al. (2016) reported that the transferred EVs could deliver IL-8 and VEGF to endothelial cells to change their angiogenic signature and thereby prolong the survival of NB4 cells. Among the different angiogenic cargos, perhaps the miR-17-192 family is the most important noncoding RNAs that could stimulate angiogenesis in acute leukemia (Doebele et al., 2010). Through suppressing integrin A5 in human umbilical vein endothelial cells (HUVECs), it has been proposed that miR-92a, one of the members of the miR-17-92 family, could increase the density of microvesicles in the BM microenvironment (Xin et al., 2017). Apart from direct delivery of angiogenic factors, leukemic cells could also induce hypoxia in endothelial cells through EVs carrying HIF-1 $\alpha$. In response to hypoxia, endothelial cells activate their angiogenic properties, so that the new vessels might bring enough supplies of oxygen to the cells (Park et al., 2010). In ALL cases, the delivered exo-miR-181 could also be transferred into endothelial cells and enforce these cells to produce VEGF to enhance angiogenesis (Patel et al., 2016). Overall, these findings shed light on the importance of EVs in the progression and dissemination of myeloid leukemia cells through regulating the angiogenic process.

\section{The Role of Extracellular Vesicles in Regulating Drug-Resistance}

Constructing a leukemia-friendly environment, EVs also protect leukemic cells from anti-cancer agents. Viola et al. (2016) was the first group who have reported that the transferred TGF- $\beta$ and miR-155 from AML cells to MSCs provide a chemotherapyprotective environment for AML cells. Wojtuszkiewicz et al. (2016) realized that the chemo-sensitive AML cells could acquire the resistant phenotype through receiving $\mathrm{Bcl}-2$ containing EVs from the chemo-resistant AML cells. They proposed that EVs are communicational tools for inducing drug resistance (Wojtuszkiewicz et al., 2016). The same results were also reported 


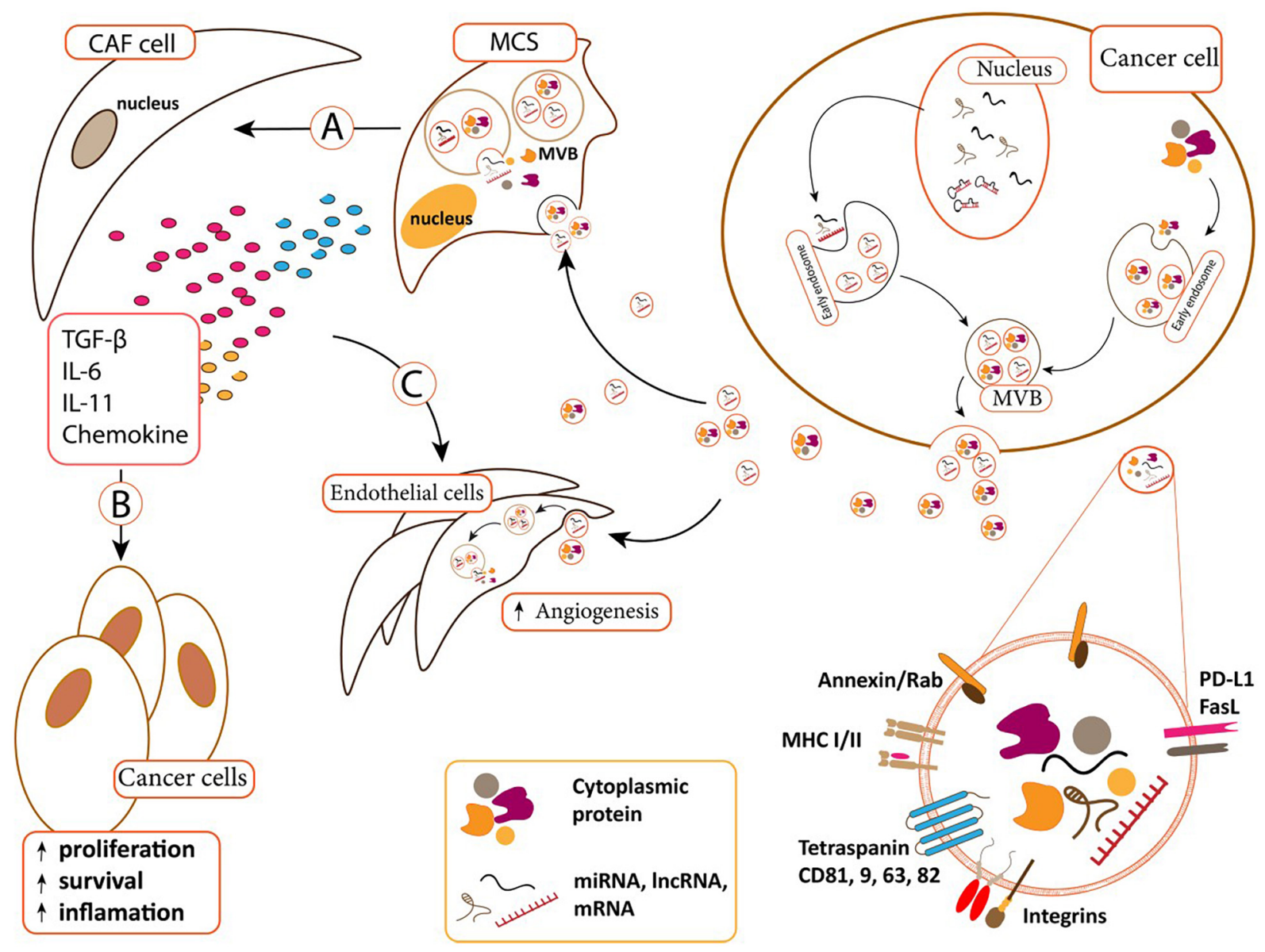

FIGURE 3 | (A) Cancer-derived exosomes can transfer to MSCs and change MSCs. MSCs differentiated to cancer-associated fibroblast (CAF) in B.M microenvironment. (B) CAFs supports cancer cells via secreted content such as TGF- $\beta$, IL-11, IL-6, and chemokine. CAF secreted contents affect cancer cells and increase cancer cell proliferation, survival. (C) Cancer derived exosomes can also uptake by endothelial cells and induce angiogenesis. CAFs secreted content can also induce angiogenesis in endothelial cells (Padua and Massagué, 2009; Mineo et al., 2012; Pickup et al., 2013; Costanza et al., 2017).

by Bouvy et al. (2017) who indicated that daunorubicin-resistant AML cells could induce drug resistance in other leukemic cells through delivering drug efflux pump multidrug resistance protein 1 (MRP-1). Aberrant delivery of the anti-apoptotic molecules/proteins to AML cells is well-studied in numerous studies and has been suggested as a mechanism to increase the survival of leukemic cells against anti-cancer agents. For example, miR-155 and miR-375 enriched EVs could be transferred from BMSCs to confer drug resistant phenotype in AML cells against tyrosine kinase inhibitors (Viola et al., 2016). MiR-19b and miR20 could induce chemo-resistance through activating TGF- $\beta$ and PI3K/Akt signaling axis (Tazzari et al., 2007). In the induction of resistance against immune-therapies, the footprint of EVs is also observed. Hong et al. (2017) suggested that AML-derived EVs could attenuate the efficacy of adoptive natural killer (NK) cell therapy by delivering inhibitory ligands that counteract the activity of NKG2D receptor on NK-92 cells. The transferred TGF- $\beta$ from AML cells into NK-92 cells reduced the expression of NKG2D in these cells through recruiting TGF $\beta$ RI/II pathway (Hong et al., 2017).
The number of studies demonstrated the role of exomiRNAs/proteins in conferring drug resistant phenotype is rare in ALL cases. It has been reported that when BMSCs absorb leukemia-derived EVs, they would be transformed into cancer associated fibroblasts (CAFs) that could evolve a protective niche against chemotherapeutic drugs (Figure 3). On the reciprocal manner, BMSCs-derived EVs could deliver galectin-3 into ALL cells to increase their drug resistance through activating NF- $\kappa B$ signaling axis (Fei et al., 2015).

\section{Extracellular Vesicles and Acute Leukemia Prognosis}

Given the importance of EVs in the pathogenesis of acute leukemia, many studies have come to a consensus that analyzing the contents of these circulating vesicles could provide a valuable perspective about the outcome of patient with leukemia. Table 2 summarized the results of several studies evaluating the prognostic value of EVs in leukemia patients. 
TABLE 2 | The correlation between leukemia-derived EVs and the outcome of patients.

\begin{tabular}{|c|c|c|}
\hline Study & Results & References \\
\hline \multicolumn{3}{|l|}{ AML } \\
\hline Bernardi et al. & $\begin{array}{l}\text { AML patients with higher exosome levels of miR-10b had shorter survival as compared to those with lower } \\
\text { levels of miR-10b. }\end{array}$ & Bernardi and Farina, 2021 \\
\hline Bernardi et al. & $\begin{array}{l}\text { The higher expression of exosome miR-532 in AML patients is associated with lower survival, suggesting } \\
\text { that miR-532 can act as an independent prognostic marker in AML. }\end{array}$ & Bernardi and Farina, 2021 \\
\hline Fang et al. & $\begin{array}{l}\text { The higher expression of miR-10b in exosomes harvested from the sera of AML patients is associated with } \\
\text { the aggressive clinical characteristics of the disease and poorer outcomes in the patients. }\end{array}$ & Fang et al., 2020 \\
\hline Jiang et al. & $\begin{array}{l}\text { The identification of miR-125b in EVs of AML patients is suggestive of elevated risk of disease relapse and } \\
\text { shorter 2-years overall survival, introducing miR-125b as an independent prognostic marker. }\end{array}$ & Jiang et al., 2018 \\
\hline Kontopoulou et al. & $\begin{array}{l}\text { Genetic analysis of EVs harvested from pediatric AML patients could be used as a tool to evaluate MRD in } \\
\text { the patients. }\end{array}$ & Kontopoulou et al., 2020 \\
\hline Chen et al. & $\begin{array}{l}\text { EVs encapsulating miR-1246 could increase the survival of leukemic stem cells (LSCs) in AML patients } \\
\text { through targeting LRIG1 and STAT-3 signaling pathway and thereby induce poor outcomes in the patients. }\end{array}$ & Chen et al., 2021 \\
\hline Bouvy et al. & $\begin{array}{l}\text { MRP-1 proteins could be delivered from chemo-resistance HL-60 cells to chemo-sensitive leukemic cells. } \\
\text { Circulating EVs containing miR-19b and miR-20a are responsible for the induction of chemo-resistance in } \\
\text { AML patients and thereby reduce their overall survival. }\end{array}$ & Bouvy et al., 2017 \\
\hline Barzegar et al. & $\begin{array}{l}\text { AML-derived EVs containing MRD proteins could induce chemo-resistance against idarubicin, suggestive of } \\
\text { the participation of EVs in the induction of poor prognosis in AML patients. }\end{array}$ & Barzegar et al., 2021 \\
\hline Viola et al. & $\begin{array}{l}\text { BMSC-derived EVs that contain miR-155 could confer drug resistance against tyrosine kinase inhibitors in } \\
\text { AML patients, reduce the opportunity of complete remission in patients. }\end{array}$ & Viola et al., 2016 \\
\hline Lin et al. & Elevated plasma exosome-derived miR-532 is associated with favorable outcomes in AML patients. & Lin et al., 2020 \\
\hline Jiang et al. & $\begin{array}{l}\text { An increased in the expression of exosome miR-125b could increase the risk of relapse in AML patients and } \\
\text { is associated with the reduced overall survival. }\end{array}$ & Jiang et al., 2018 \\
\hline Hong et al. & $\begin{array}{l}\text { The reduction in the levels of plasma EV-TGF } \beta 1 \text { protein in AML patients who received chemotherapy is } \\
\text { indicative of the favorable response to treatment and induction of long-term complete remission. Changes } \\
\text { in EV-TGF } \beta 1 \text { levels in AML patients could be considered a prognostic and risk stratifying factor. }\end{array}$ & Hong et al., 2017 \\
\hline Hornick et al. & $\begin{array}{l}\text { Elevated serum EV levels containing let-7a, miR-99b, }-146 a \text {, and }-191 \text {, is associated with poor prognosis in } \\
\text { AML patients. }\end{array}$ & Hornick et al., 2015 \\
\hline Caviano et al. & $\begin{array}{l}\text { EV-derived miR-155 is a well-known independent prognostic factor for AML patients. The level of this } \\
\text { miRNA correlates with the number of WBCs and complex karyotypes in patients. }\end{array}$ & Caivano et al., 2017 \\
\hline \multicolumn{3}{|l|}{ ALL } \\
\hline Egyed et al. & $\begin{array}{l}\text { The elevation in EV containing miR-181a could be an indicator for CNS involvement for the pediatric } \\
\text { patients with ALL. }\end{array}$ & Egyed et al., 2020 \\
\hline Labib et al. & $\begin{array}{l}\text { The upregulation of extracellular miR-22 in pediatric ALL is associated with poor prognosis and shorter } \\
\text { overall survival. }\end{array}$ & Labib et al., 2017 \\
\hline Rzepiel et al. & $\begin{array}{l}\text { Detetction of miR-128-3p and miR-222-3p in blood of ALL patients could be indicator of MRD and thus far } \\
\text { these circulating miRNAs could be considered a prognostic maker for ALL patients. }\end{array}$ & Rzepiel et al., 2019 \\
\hline
\end{tabular}

\section{CHALLENGES OF EXTRACELLULAR VESICLES IN THE CLINICAL APPLICATION FOR ACUTE LEUKEMIA}

Given the importance of EVs in the pathogenesis of leukemia and based on the number of reports suggesting the benefits of evaluating EVs contents for early diagnosis or predicting the outcome of patients, it could be known that EVs are promising circulating biomarkers. However, the story is not as simple as it looks. The study of EVs as biomarkers in clinical approaches is still a new field, and no standard methods have been established yet for the proper enrichment and isolation of these circulating vesicles. The diversity in the protocols used for EVs isolation, enrichment, and measurement leads to the fact that in many cases, the results of studies are not comparable with each other, and this may have a negative effect on the validation of the results (Lane et al., 2018). For example, for EVs isolation, based on the available equipment and materials, methods such as differential ultracentrifugation, density gradient ultracentrifugation, polymer-facilitated precipitation, immuneaffinity isolation and, size exclusion chromatography (SEC) are used. Among them, differential ultracentrifugation is considered a gold standard method for EV isolation (Théry et al., 2006). Despite the great popularity, ultracentrifugation could lead to vesicle aggregation and contamination of the protein contents of EVs (Baranyai et al., 2015; Nordin et al., 2015). Moreover, the variability in the methods in evaluating pelleting efficiency has led to the discrepancy in results obtained from different studies. To tackle the contamination problem, density gradient ultracentrifugation was developed (Kalra et al., 2013; Choi and Gho, 2015). However, the difficulty in the procedure of this method and the risk of loss of samples have muted the enthusiasm in employing density gradient ultracentrifugation (Muller et al., 2014). For the polymer-facilitated precipitation and the immuneaffinity isolation, the poor purity of isolated EVs and the lack of proper antibodies have been claimed as the main factors that 
could restrict the efficacy of the methods (Van Deun et al., 2014; Lane et al., 2018).

The diversity in the isolation techniques could not only produce variability in the results but also influence the precise molecular characterization of EVs. The high purity of EVs and ability to distinct the EV-derived proteins/nucleic acids from non-EV sources are essential for the proper characterization of EVs. Apart from this, thus far, several techniques, including transmission electron microscopy (TEM), dynamic light scattering (DLS), nanoparticle tracking analysis (NTA), flow cytometry, and tunable resistive pulse sensing (TRPS) are used for the physical characterization of EVs. Although effective, each of these techniques also have some limitations and complications. Problems such as vesicle shrinkage and the limited number of vesicles that could be analyzed by TEM have made flow cytometry superior to TEM which is currently a gold standard method (Van der Pol et al., 2014; Erdbrügger and Lannigan, 2016). However, the application of flow cytometry is not without practical limitation, as the small size of EVs and their low refractive index result in improper scattering of the vesicles by flow cytometry (Van Der Pol et al., 2012; Nolan, 2015). The labeling of EVs with protein-dye was not successful in tackling this problem, as EVs possess a restricted amount of target molecules (Welsh et al., 2017). A similar problem could be seen in studies using DLS and NTA for the molecular characterization of EVs. Although these techniques are rapid, i.e., NTA could measure the contents of thousands of single EVs in less than a few minutes (Bi et al., 2021) and could analyze bulk samples, only larger particles with the ability to scatter the greater amount of light signal could be characterized by these methods (Anderson et al., 2013; Lane et al., 2018). The nature of TRPS is different from other methods and this nonoptical measurement technique uses the electrical impedance for analyzing the physical characterization of EVs. However, the necessity of an expert user to operate this technique has made it difficult to use TRPS for the characterization of EVs (Lane et al., 2018). It should be noted that both qRT-PCR and Western blotting analysis for evaluating the miRNA/RNA and protein content of EVs could also be affected by the methods that are used for the isolation of EVs. Taken together, all mentioned limitations suggest the necessity of an appropriate protocol for EV handling and measurement. The more accurate the methods, the faster EVs could be employed in clinical settings for risk stratifying patients.

\section{CAN EXTRACELLULAR VESICLES MEET THE CLINICAL CHALLENGES FOR RISK STRATIFICATION IN PATIENTS WITH ACUTE LEUKEMIA?}

The application of EVs in risk stratification for patients with acute leukemia is beneficial, though it has a long way to be achievable. First, as mentioned earlier, due to the limitation of all mentioned isolation techniques in distinguishing exosomes from micro-vesicles, both of these cellular components referred to as circulating vesicles, which limits our understanding of the property of these circular vesicles. The less we know about the contents of circulating EVs, the longer it might take for receiving approval EVs as a diagnostic tool in clinical application (Witwer et al., 2013). Moreover, some studies revealed that the level of circulating EVs could be affected by numerous factors, and thereby, EVs varied based on the time of sample collection (Pickup et al., 2013). This finding threatens the value of $\mathrm{EVs}$ in the clinical application and prioritizes the importance of an optimized protocol for the collection, isolation, and storage of EVs. Additionally, many studies have thus far evaluated the efficacy and the value of EVs in in vitro analysis. So, experiments conducting on patient's samples are required to achieve better results and provide a wider perspective about the application of EVs as a prognostic factor in patients with acute leukemia.

\section{CONCLUSION}

From the first description of EVs in the samples of patients with leukemia, there is no doubt that these tiny circulating vesicles play fundamental roles in leukemogenesis, cell proliferation, survival, and also angiogenesis. The components of EVs conveniently alter the structure of BM in the way that it protects leukemic cells from either the adaptive arm of the immune system or anti-cancer agents. Thus far, many studies are focusing on the importance and value of EVs in determining the outcome of patients with leukemia; however, a long way should be passed to reach the best results. As it was mentioned earlier, according to the size of EVs, three classes of these circulating vesicles have been identified in body fluids and for sure, each of them may participate in some specific biological processes. However, due to the size overlap and the disability of the current isolation and characterization technologies, it is impossible to distinguish these vesicles from each other and analyze their cargos individually. Moreover, many of the results published in this area are conflicting and incomparable, as each research group might use different techniques for the collection, processing, and storage of EVs. Given these limitations, it seems that more issues should be addressed before EVs could enter into the clinical application for acute leukemia. Nevertheless, the journey of EVs in leukemia is still mesmerizing.

\section{AUTHOR CONTRIBUTIONS}

MI: conceptualization, literature survey, figure designing, review structure, writing review and editing, and references collection. $\mathrm{ZH}$ : conceptualization, literature survey, review structure, writing review and editing, and references collection. FJ: literature survey, writing review, and references collection. AH and AG: critical review and editing. Y-DL: formating and reference collection. 
LJ: conceptualization, literature survey, writing review and editing, references collection, tables preparation, supervision, and correspondence. Z-SC: critical review and editing, correspondence, and supervision. All authors contributed to the article and approved the submitted version.

\section{REFERENCES}

Akers, J. C., Gonda, D., Kim, R., Carter, B. S., and Chen, C. C. (2013). Biogenesis of extracellular vesicles (EV): exosomes, microvesicles, retrovirus-like vesicles, and apoptotic bodies. J. Neuro Oncol. 113, 1-11. doi: 10.1007/s11060-013-1084-8

Alemdehy, M. F., de Looper, H., Kavelaars, F., Sanders, M., Hoogenboezem, R., Löwenberg, B., et al. (2016). MicroRNA-155 induces AML in combination with the loss of C/EBPA in mice. Leukemia 30, 2238-2241. doi: 10.1038/leu.2016.171

Anderson, W., Kozak, D., Coleman, V. A., Jämting, ÅK., and Trau, M. (2013). A comparative study of submicron particle sizing platforms: accuracy, precision and resolution analysis of polydisperse particle size distributions. J. Colloid Interface Sci. 405, 322-330. doi: 10.1016/j.jcis.2013.02.030

Baranyai, T., Herczeg, K., Onódi, Z., Voszka, I., Módos, K., Marton, N., et al. (2015). Isolation of exosomes from blood plasma: qualitative and quantitative comparison of ultracentrifugation and size exclusion chromatography methods. PloS One 10:e0145686. doi: 10.1371/journal.pone.0145686

Barzegar, M., Allahbakhshian Farsani, M., Amiri, V., Mohammadi, S., Shahsavan, S., Mirzaeian, A., et al. (2021). AML-derived extracellular vesicles confer de novo chemoresistance to leukemic myeloblast cells by promoting drug export genes expression and ROS inhibition. Iran. J. Pharm. Res. 20, 384-397.

Bassan, R., and Hoelzer, D. (2011). Modern therapy of acute lymphoblastic leukemia. J. Clin. Oncol. 29, 532-543. doi: 10.1200/jco.2010.30.1382

Battula, V. L., Le, P. M., Sun, J. C., Nguyen, K., Yuan, B., Zhou, X., et al. (2017). AML-induced osteogenic differentiation in mesenchymal stromal cells supports leukemia growth. JCI Insight 2:e90036.

Bernardi, S., and Farina, M. (2021). Exosomes and extracellular vesicles in myeloid neoplasia: The multiple and complex roles played by these "Magic Bullets". Biology 10:105. doi: 10.3390/biology10020105

Bernardi, S., Zanaglio, C., Farina, M., Polverelli, N., Malagola, M., and Russo, D. (2021). dsDNA from extracellular vesicles (EVs) in adult AML. Ann. Hematol. 100, 1355-1356. doi: 10.1007/s00277-020-04109-z

Bi, J., Pu, Y., and Yu, X. (2021). Exosomal circ_0004136 enhances the progression of pediatric acute myeloid leukemia depending on the regulation of miR570-3p/TSPAN3 axis. Anticancer Drugs 32, 802-811. doi: 10.1097/cad. 0000000000001068

Bi, L., Sun, L., Jin, Z., Zhang, S., and Shen, Z. (2018). MicroRNA-10a/b are regulators of myeloid differentiation and acute myeloid leukemia. Oncol. Lett. $15,5611-5619$.

Bosshard, R., O’Reilly, K., Ralston, S., Chadda, S., and Cork, D. (2018). Systematic reviews of economic burden and health-related quality of life in patients with acute myeloid leukemia. Cancer Treatment Rev. 69, 224-232. doi: 10.1016/j. ctrv.2018.07.005

Bousquet, M., Harris, M. H., Zhou, B., and Lodish, H. F. (2010). MicroRNA miR125b causes leukemia. Proc. Natl. Acad. Sci. 107, 21558-21563. doi: 10.1073/ pnas. 1016611107

Bouvy, C., Wannez, A., Laloy, J., Chatelain, C., and Dogné, J. M. (2017). Transfer of multidrug resistance among acute myeloid leukemia cells via extracellular vesicles and their microRNA cargo. Leukemia Res. 62, 70-76. doi: 10.1016/j. leukres.2017.09.014

Caivano, A., La Rocca, F., Simeon, V., Girasole, M., Dinarelli, S., Laurenzana, I., et al. (2017). MicroRNA-155 in serum-derived extracellular vesicles as a potential biomarker for hematologic malignancies-a short report. Cell. Oncol. 40, 97-103. doi: 10.1007/s13402-016-0300-x

Chen, L., Guo, Z., Zhou, Y., Ni, J., Zhu, J., Fan, X., et al. (2021). microRNA-1246containing extracellular vesicles from acute myeloid leukemia cells promote the survival of leukemia stem cells via the LRIG1-meditated STAT3 pathway. Aging (Albany NY). 13:13644. doi: 10.18632/aging.202893

Choi, D.-S., and Gho, Y. S. (2015). Isolation of extracellular vesicles for proteomic profiling. Methods Mol. Biol. 1295, 167-177. doi: 10.1007/978-1-4939-2 550-6_14

\section{FUNDING}

We thank the partially support from the Startup Foundation for Doctors of The First Affiliated Hospital of Gannan Medical University, China.

Chu, Y. L., Li, H., Ng, P. L. A., Kong, S. T., Zhang, H., Lin, Y., et al. (2020). The potential of circulating exosomal RNA biomarkers in cancer. Expert Rev.Mol. Diagn. 20, 665-678.

Cocozza, F., Grisard, E., Martin-Jaular, L., Mathieu, M., and Thery, C. (2020). SnapShot: extracellular vesicles. Cell 182:262. doi: 10.1016/j.cell.2020.04.054

Corrado, C., Raimondo, S., Saieva, L., Flugy, A. M., De Leo, G., and Alessandro, R. (2014). Exosome-mediated crosstalk between chronic myelogenous leukemia cells and human bone marrow stromal cells triggers an interleukin 8-dependent survival of leukemia cells. Cancer Lett. 348, 71-76. doi: 10.1016/j.canlet.2014. 03.009

Costanza, B., Umelo, I. A., Bellier, J., Castronovo, V., and Turtoi, A. (2017). Stromal modulators of TGF- $\beta$ in cancer. J. Clin. Med. 6:7. doi: 10.3390/jcm6010007

Crenshaw, B. J., Sims, B., and Matthews, Q. L. (2018). "Biological function of exosomes as diagnostic markers and therapeutic delivery vehicles in carcinogenesis and infectious diseases," in Nanomedicines, ed. M. A. Farrukh (London: IntechOpen).

D'Souza-Schorey, C., and Schorey, J. S. (2018). Regulation and mechanisms of extracellular vesicle biogenesis and secretion. Essays Biochem. 62, 125-133. doi: 10.1042/ebc20170078

Da, M., Jiang, H., Xie, Y., Jin, W., and Han, S. (2021). The biological roles of exosomal long non-coding RNAs in cancers. OncoTargets Ther. 14, 271-287. doi: $10.2147 /$ ott.s281175

Doebele, C., Bonauer, A., Fischer, A., Scholz, A., Reiss, Y., Urbich, C., et al. (2010). Members of the microRNA-17-92 cluster exhibit a cell-intrinsic antiangiogenic function in endothelial cells. Blood 115, 4944-4950. doi: 10.1182/blood-201001-264812

Doyle, L. M., and Wang, M. Z. (2019). Overview of extracellular vesicles, their origin, composition, purpose, and methods for exosome isolation and analysis. Cells 8:727. doi: 10.3390/cells8070727

Egyed, B., Kutszegi, N., Sági, J. C., Gézsi, A., Rzepiel, A., Visnovitz, T., et al. (2020). MicroRNA-181a as novel liquid biopsy marker of central nervous system involvement in pediatric acute lymphoblastic leukemia. J. Transl. Med. $18,1-12$.

Erdbrügger, U., and Lannigan, J. (2016). Analytical challenges of extracellular vesicle detection: A comparison of different techniques. Cytometry Part A. 89, 123-134. doi: 10.1002/cyto.a.22795

Fang, Y., Garnier, D., Lee, T. H., D’Asti, E., Montermini, L., Meehan, B., et al. (2016). PML-RARa modulates the vascular signature of extracellular vesicles released by acute promyelocytic leukemia cells. Angiogenesis 19, 25-38. doi: 10.1007/s10456-015-9486-1

Fang, Z., Wang, X., Wu, J., Xiao, R., and Liu, J. (2020). High serum extracellular vesicle miR-10b expression predicts poor prognosis in patients with acute myeloid leukemia. Cancer Biomark. 27, 1-9. doi: 10.3233/cbm-190211

Fei, F., Joo, E. J., Tarighat, S. S., Schiffer, I., Paz, H., Fabbri, M., et al. (2015). B-cell precursor acute lymphoblastic leukemia and stromal cells communicate through Galectin-3. Oncotarget 6:11378. doi: 10.18632/oncotarget. 3409

Fong, M. Y., Zhou, W., Liu, L., Alontaga, A. Y., Chandra, M., Ashby, J., et al. (2015). Breast-cancer-secreted miR-122 reprograms glucose metabolism in premetastatic niche to promote metastasis. Nat. Cell Biol. 17, 183-194. doi: $10.1038 /$ ncb3094

Forman, J. J., Legesse-Miller, A., and Coller, H. A. (2008). A search for conserved sequences in coding regions reveals that the let-7 microRNA targets Dicer within its coding sequence. Proc. Natl. Acad. Sci. 105, 14879-14884. doi: 10. 1073/pnas.0803230105

Groot, M., and Lee, H. (2020). Sorting mechanisms for MicroRNAs into extracellular vesicles and their associated diseases. Cells 9:1044. doi: 10.3390/ cells 9041044

Gurunathan, S., Kang, M.-H., Qasim, M., Khan, K., and Kim, J.-H. (2021). Biogenesis, membrane trafficking, functions, and next generation 
nanotherapeutics medicine of extracellular vesicles. Int. J. Nanomed. 16:3357. doi: 10.2147/ijn.s310357

Han, J., Lee, Y., Yeom, K.-H., Kim, Y.-K., Jin, H., and Kim, V. N. (2004). The Drosha-DGCR8 complex in primary microRNA processing. Genes Dev. 18, 3016-3027. doi: 10.1101/gad.1262504

Haque, S., and Vaiselbuh, S. R. (2020). Silencing of exosomal miR-181a reverses pediatric acute lymphocytic leukemia cell proliferation. Pharmaceuticals (Basel) 13:241. doi: 10.3390/ph13090241

He, C., Zheng, S., Luo, Y., and Wang, B. (2018). Exosome theranostics: biology and translational medicine. Theranostics 8, 237-255. doi: 10.7150/thno.21945

Hemler, M. E. (2005). Tetraspanin functions and associated microdomains. Nat. Rev. Mol. Cell Biol. 6, 801-811. doi: 10.1038/nrm1736

Hessvik, N. P., and Llorente, A. (2018). Current knowledge on exosome biogenesis and release. Cell. Mol. Life Sci. 75, 193-208. doi: 10.1007/s00018-017-2595-9

Hong, C.-S., Sharma, P., Yerneni, S. S., Simms, P., Jackson, E. K., Whiteside, T. L., et al. (2017). Circulating exosomes carrying an immunosuppressive cargo interfere with cellular immunotherapy in acute myeloid leukemia. Sci. Rep. 7, 14684.

Hornick, N. I., Doron, B., Abdelhamed, S., Huan, J., Harrington, C. A., Shen, R., et al. (2016). AML suppresses hematopoiesis by releasing exosomes that contain microRNAs targeting c-MYB. Sci. Signal. 9:ra88. doi: 10.1126/scisignal. aaf2797

Hornick, N. I., Huan, J., Doron, B., Goloviznina, N. A., Lapidus, J., Chang, B. H., et al. (2015). Serum exosome microRNA as a minimally-invasive early biomarker of AML. Sci. Rep. 5, 1-12.

Huan, J., Hornick, N. I., Shurtleff, M. J., Skinner, A. M., Goloviznina, N. A., Roberts, C. T., et al. (2013). RNA trafficking by acute myelogenous leukemia exosomes. Cancer Res. 73, 918-929. doi: 10.1158/0008-5472.can-12-2184

Huang, T., and Deng, C. X. (2019). Current progresses of exosomes as cancer diagnostic and prognostic biomarkers. Int. J. Biol. Sci. 15, 1-11. doi: 10.7150/ ijbs. 27796

Hunger, S. P., and Mullighan, C. G. (2015). Acute lymphoblastic leukemia in children. New Eng. J. Med. 373, 1541-1552.

Huntzinger, E., and Izaurralde, E. (2011). Gene silencing by microRNAs: contributions of translational repression and mRNA decay. Nat. Rev. Genet. 12, 99-110. doi: 10.1038/nrg2936

Ibrahim, A., and Marbán, E. (2016). Exosomes: fundamental biology and roles in cardiovascular physiology. Annu. Rev. Physiol. 78, 67-83. doi: 10.1146/ annurev-physiol-021115-104929

Ingenito, F., Roscigno, G., Affinito, A., Nuzzo, S., Scognamiglio, I., Quintavalle, C., et al. (2019). The role of Exo-miRNAs in cancer: A focus on therapeutic and diagnostic applications. Int. J. Mol. Sci. 20:4687. doi: 10.3390/ijms2019 4687

Jadli, A. S., Ballasy, N., Edalat, P., and Patel, V. B. (2020). Inside(sight) of tiny communicator: exosome biogenesis, secretion, and uptake. Mol. Cell. Biochem. 467, 77-94. doi: 10.1007/s11010-020-03703-z

Jansen, F. H., Krijgsveld, J., van Rijswijk, A., van den Bemd, G. J., van den Berg, M. S., van Weerden, W. M., et al. (2009). Exosomal secretion of cytoplasmic prostate cancer xenograft-derived proteins. Mol. Cell. Proteomics 8, 1192-1205. doi: 10.1074/mcp.m800443-mcp200

Jeppesen, D. K., Fenix, A. M., Franklin, J. L., Higginbotham, J. N., Zhang, Q., Zimmerman, L. J., et al. (2019). Reassessment of exosome composition. Cell 177, 428-445. doi: 10.1016/j.cell.2019.02.029

Ji, D., He, Y., Lu, W., Rong, Y., Li, F., Huang, X., et al. (2021). Smallsized extracellular vesicles (EVs) derived from acute myeloid leukemia bone marrow mesenchymal stem cells transfer miR-26a-5p to promote acute myeloid leukemia cell proliferation, migration, and invasion. Hum. Cell. 34, 965-976. doi: 10.1007/s13577-021-00501-7

Jiang, L., Deng, T., Wang, D., and Xiao, Y. (2018). Elevated serum exosomal miR$125 \mathrm{~b}$ level as a potential marker for poor prognosis in intermediate-risk acute myeloid leukemia. Acta Haematol. 140, 183-192. doi: 10.1159/000491584

Johnson, S. M., Dempsey, C., Chadwick, A., Harrison, S., Liu, J., Di, Y., et al. (2016). Metabolic reprogramming of bone marrow stromal cells by leukemic extracellular vesicles in acute lymphoblastic leukemia. Blood 128, 453-456. doi: 10.1182/blood-2015-12-688051

Juan, T., and Furthauer, M. (2018). Biogenesis and function of ESCRT-dependent extracellular vesicles. Semin. Cell Dev. Biol. 74, 66-77. doi: 10.1016/j.semcdb. 2017.08.022
Kajimoto, T., Okada, T., Miya, S., Zhang, L., and Nakamura, S. (2013). Ongoing activation of sphingosine 1-phosphate receptors mediates maturation of exosomal multivesicular endosomes. Nat. Commun. 4:2712.

Kalluri, R., and LeBleu, V. S. (2020). The biology, function, and biomedical applications of exosomes. Science 367:eaau6977. doi: 10.1126/science.aau6977

Kalra, H., Adda, C. G., Liem, M., Ang, C. S., Mechler, A., Simpson, R. J., et al. (2013). Comparative proteomics evaluation of plasma exosome isolation techniques and assessment of the stability of exosomes in normal human blood plasma. Proteomics 13, 3354-3364. doi: 10.1002/pmic.201300282

Kim, Y. S., Ahn, J. S., Kim, S., Kim, H. J., Kim, S. H., and Kang, J. S. (2018). The potential theragnostic (diagnostic+therapeutic) application of exosomes in diverse biomedical fields. Korean J. Physiol. Pharmacol. 22, 113-125. doi: 10.4196/kjpp.2018.22.2.113

King, H. W., Michael, M. Z., and Gleadle, J. M. (2012). Hypoxic enhancement of exosome release by breast cancer cells. BMC Cancer 12:421. doi: 10.1186/14712407-12-421

Kontopoulou, E., Strachan, S., Reinhardt, K., Kunz, F., Walter, C., Walkenfort, B., et al. (2020). Evaluation of dsDNA from extracellular vesicles (EVs) in pediatric AML diagnostics. Ann. Hematol. 99, 459-475. doi: 10.1007/s00277-01903866-w

Kosaka, N., Iguchi, H., Yoshioka, Y., Takeshita, F., Matsuki, Y., and Ochiya, T. (2010). Secretory mechanisms and intercellular transfer of microRNAs in living cells. J. Biol. Chem. 285, 17442-17452. doi: 10.1074/jbc.m110.107821

Kumar, B., Garcia, M., Weng, L., Jung, X., Murakami, J., Hu, X., et al. (2018). Acute myeloid leukemia transforms the bone marrow niche into a leukemiapermissive microenvironment through exosome secretion. Leukemia 32, 575587. doi: 10.1038/leu.2017.259

Labib, H. A., Elantouny, N. G., Ibrahim, N. F., and Alnagar, A. A. (2017). Upregulation of microRNA-21 is a poor prognostic marker in patients with childhood B cell acute lymphoblastic leukemia. Hematology 22, 392-397. doi: 10.1080/10245332.2017.1292204

Lane, R., Korbie, D., Hill, M., and Trau, M. (2018). Extracellular vesicles as circulating cancer biomarkers: opportunities and challenges. Clin. Transl. Med. 7, 1-11. doi: 10.1007/978-94-007-7742-2_38-1

Li, B., Antonyak, M. A., Zhang, J., and Cerione, R. A. (2012). RhoA triggers a specific signaling pathway that generates transforming microvesicles in cancer cells. Oncogene 31, 4740-4749. doi: 10.1038/onc.2011.636

Li, C., Gao, Q., Wang, M., and Xin, H. (2021). LncRNA SNHG1 contributes to the regulation of acute myeloid leukemia cell growth by modulating miR-4893p/SOX12/Wnt/ß-catenin signaling. J. Cell. Physiol. 236, 653-663. doi: 10.1002/ jcp. 29892

Li, L., Zhu, D., Huang, L., Zhang, J., Bian, Z., Chen, X., et al. (2012). Argonaute 2 complexes selectively protect the circulating microRNAs in cell-secreted microvesicles. PLoS One 7:e46957. doi: 10.1371/journal.pone.0046957

Lin, X., Ling, Q., Lv, Y., Ye, W., Huang, J., Li, X., et al. (2020). Plasma exosomederived microRNA-532 as a novel predictor for acute myeloid leukemia. Cancer Biomark. 28, 151-158. doi: 10.3233/cbm-191164

Litwińska, Z., Łuczkowska, K., and Machaliński, B. (2019). Extracellular vesicles in hematological malignancies. Leukemia Lymphoma 60, 29-36. doi: 10.1080/ 10428194.2018.1459606

Liu, Y., Shi, K., Chen, Y., Wu, X., Chen, Z., Cao, K., et al. (2021). Exosomes and their role in cancer progression. Front. Oncol. 11:639159. doi: 10.3389/fonc. 2021.639159

Luger, S. M. (2017). How can one optimize induction therapy in AML? Best Pract. Res. Clin. Haematol. 30, 301-305. doi: 10.1016/j.beha.2017.10.001

Lv, M., Zhu, S., Peng, H., Cheng, Z., Zhang, G., and Wang, Z. (2021). B-cell acute lymphoblastic leukemia-related microRNAs: uncovering their diverse and special roles. Am. J. Cancer Res. 11:1104.

Mashouri, L., Yousefi, H., Aref, A. R., Ahadi, A. M., Molaei, F., and Alahari, S. K. (2019). Exosomes: composition, biogenesis, and mechanisms in cancer metastasis and drug resistance. Mol. Cancer 18:75.

McKenzie, A. J., Hoshino, D., Hong, N. H., Cha, D. J., Franklin, J. L., Coffey, R. J., et al. (2016). KRAS-MEK signaling controls Ago2 sorting into exosomes. Cell Rep. 15, 978-987. doi: 10.1016/j.celrep.2016.03.085

Mineo, M., Garfield, S. H., Taverna, S., Flugy, A., De Leo, G., Alessandro, R., et al. (2012). Exosomes released by K562 chronic myeloid leukemia cells promote angiogenesis in a Src-dependent fashion. Angiogenesis 15, 33-45. doi: 10.1007/ s10456-011-9241-1 
Muller, L., Hong, C.-S., Stolz, D. B., Watkins, S. C., and Whiteside, T. L. (2014). Isolation of biologically-active exosomes from human plasma. J. Immunol. Methods 411, 55-65. doi: 10.1016/j.jim.2014.06.007

Muralidharan-Chari, V., Clancy, J., Plou, C., Romao, M., Chavrier, P., Raposo, G., et al. (2009). ARF6-regulated shedding of tumor cellderived plasma membrane microvesicles. Curr. Biol. 19, 1875-1885. doi: 10.1016/j.cub.2009.09.059

Nolan, J. P. (2015). Flow cytometry of extracellular vesicles: potential, pitfalls, and prospects. Curr. Prot. cytometry 73, 13.14.1-13.14.16.

Nordin, J. Z., Lee, Y., Vader, P., Mäger, I., Johansson, H. J., Heusermann, W., et al. (2015). Ultrafiltration with size-exclusion liquid chromatography for high yield isolation of extracellular vesicles preserving intact biophysical and functional properties. Nanomedicine 11, 879-883. doi: 10.1016/j.nano.2015. 01.003

Nunez, R., Sancho-Martinez, S., Novoa, J., and Lopez-Hernandez, F. (2010). Apoptotic volume decrease as a geometric determinant for cell dismantling into apoptotic bodies. Cell Death Differ. 17, 1665-1671. doi: 10.1038/cdd. 2010.96

O’Brien, K., Rani, S., Corcoran, C., Wallace, R., Hughes, L., Friel, A. M., et al. (2013). Exosomes from triple-negative breast cancer cells can transfer phenotypic traits representing their cells of origin to secondary cells. Eur. J. Cancer 49, 1845-1859. doi: 10.1016/j.ejca.2013.01.017

O’Brien, J., Hayder, H., Zayed, Y., and Peng, C. (2018). Overview of microRNA biogenesis, mechanisms of actions, and circulation. Front. Endocrinol. 9:402. doi: $10.3389 /$ fendo.2018.00402

Okada, C., Yamashita, E., Lee, S. J., Shibata, S., Katahira, J., Nakagawa, A., et al. (2009). A high-resolution structure of the pre-microRNA nuclear export machinery. Science 326, 1275-1279. doi: 10.1126/science.1178705

Ørom, U. A., Nielsen, F. C., and Lund, A. H. (2008). MicroRNA-10a binds the $5^{\prime}$ UTR of ribosomal protein mRNAs and enhances their translation. Mol. Cell 30, 460-471. doi: 10.1016/j.molcel.2008.05.001

Ostrowski, M., Carmo, N. B., Krumeich, S., Fanget, I., Raposo, G., Savina, A., et al. (2010). Rab27a and Rab27b control different steps of the exosome secretion pathway. Nat. Cell Biol. 12, 19-30. doi: 10.1038/ncb2000

Padua, D., and Massagué, J. (2009). Roles of TGFß in metastasis. Cell Res. 19, $89-102$.

Park, E.-H., Lee, H., Won, Y.-J., Ju, H. Y., Oh, C.-M., Ingabire, C., et al. (2015). Nationwide statistical analysis of myeloid malignancies in Korea: incidence and survival rate from 1999 to 2012. Blood Res. 50:204. doi: 10.5045/br.2015.50. 4.204

Park, J. E., Tan, H. S., Datta, A., Lai, R. C., Zhang, H., Meng, W., et al. (2010). Hypoxic tumor cell modulates its microenvironment to enhance angiogenic and metastatic potential by secretion of proteins and exosomes. Mol. Cell. proteomics 9, 1085-1099. doi: 10.1074/mcp.m900381-mcp200

Parolini, I., Federici, C., Raggi, C., Lugini, L., Palleschi, S., De Milito, A., et al. (2009). Microenvironmental $\mathrm{pH}$ is a key factor for exosome traffic in tumor cells. J. Biol. Chem. 284, 34211-34222. doi: 10.1074/jbc.m109. 041152

Patel, S. J., Darie, C. C., and Clarkson, B. D. (2016). Exosome mediated growth effect on the non-growing pre-B acute lymphoblastic leukemia cells at low starting cell density. Am. J. Transl. Res. 8:3614.

Pegtel, D. M., and Gould, S. J. (2019). Exosomes. Annu. Rev. Biochem. 88, 487-514. doi: 10.1016/b978-0-12-816053-4.00021-3

Pickup, M., Novitskiy, S., and Moses, H. L. (2013). The roles of TGF $\beta$ in the tumour microenvironment. Nat. Rev. Cancer. 13, 788-799.

Pols, M. S., and Klumperman, J. (2009). Trafficking and function of the tetraspanin CD63. Exp. Cell Res. 315, 1584-1592. doi: 10.1016/j.yexcr.2008.09.020

Rahbarghazi, R., Jabbari, N., Sani, N. A., Asghari, R., Salimi, L., Kalashani, S. A., et al. (2019). Tumor-derived extracellular vesicles: reliable tools for Cancer diagnosis and clinical applications. Cell Commun. Signal. 17:73.

Raposo, G., and Stoorvogel, W. (2013). Extracellular vesicles: exosomes, microvesicles, and friends. J. Cell Biol. 200, 373-383. doi: 10.1083/jcb. 201211138

Roccaro, A. M., Sacco, A., Maiso, P., Azab, A. K., Tai, Y.-T., Reagan, M., et al. (2013). BM mesenchymal stromal cell-derived exosomes facilitate multiple myeloma progression. J. Clin. Invest. 123, 1542-1555. doi: 10.1172/jci6 6517
Rzepiel, A., Kutszegi, N., Gézsi, A., Sági, J. C., Egyed, B., Péter, G., et al. (2019). Circulating microRNAs as minimal residual disease biomarkers in childhood acute lymphoblastic leukemia. J. Transl. Med. 17, 1-16.

Santangelo, L., Giurato, G., Cicchini, C., Montaldo, C., Mancone, C., Tarallo, R., et al. (2016). The RNA-binding protein SYNCRIP is a component of the hepatocyte exosomal machinery controlling microRNA sorting. Cell Rep. 17, 799-808. doi: 10.1016/j.celrep.2016.09.031

Selim, A. G., and Moore, A. S. (2018). Molecular minimal residual disease monitoring in acute myeloid leukemia: challenges and future directions. J. Mol. Diagn. 20, 389-397. doi: 10.1016/j.jmoldx.2018.03.005

Skog, J., Würdinger, T., Van Rijn, S., Meijer, D. H., Gainche, L., Curry, W. T., et al. (2008). Glioblastoma microvesicles transport RNA and proteins that promote tumour growth and provide diagnostic biomarkers. Nat. Cell Biol. 10, 1470-1476. doi: $10.1038 /$ ncb1800

Skryabin, G. O., Komelkov, A. V., Savelyeva, E. E., and Tchevkina, E. M. (2020). Lipid rafts in exosome biogenesis. Biochem. Biokhimiia 85, 177-191. doi: 10. $1134 / \mathrm{s} 0006297920020054$

Tazzari, P. L., Cappellini, A., Ricci, F., Evangelisti, C., Papa, V., Grafone, T., et al. (2007). Multidrug resistance-associated protein 1 expression is under the control of the phosphoinositide 3 kinase/Akt signal transduction network in human acute myelogenous leukemia blasts. Leukemia 21, 427-438. doi: 10.1038/sj.leu.2404523

Terwilliger, T., and Abdul-Hay, M. (2017). Acute lymphoblastic leukemia: a comprehensive review and 2017 update. Blood Cancer J. 7:e577. doi: 10.1038/ bcj. 2017.53

Théry, C., Amigorena, S., Raposo, G., and Clayton, A. (2006). Isolation and characterization of exosomes from cell culture supernatants and biological fluids. Curr. Protoc. cell Biol. 30:3.22.

Tian, Y., Ma, L., Gong, M., Su, G., Zhu, S., Zhang, W., et al. (2018). Protein profiling and sizing of extracellular vesicles from colorectal cancer patients via flow cytometry. ACS Nano 12, 671-680. doi: 10.1021/acsnano.7b07782

Valinezhad Orang, A., Safaralizadeh, R., and Kazemzadeh-Bavili, M. (2014). Mechanisms of miRNA-mediated gene regulation from common downregulation to mRNA-specific upregulation. Int. J. Genomics 2014:970607.

Van der Pol, E., Coumans, F., Grootemaat, A., Gardiner, C., Sargent, I., Harrison, P., et al. (2014). Particle size distribution of exosomes and microvesicles determined by transmission electron microscopy, flow cytometry, nanoparticle tracking analysis, and resistive pulse sensing. J. Thromb. Haemost. 12, 11821192. doi: $10.1111 /$ jth. 12602

Van Der Pol, E., Van Gemert, M., Sturk, A., Nieuwland, R., and Van Leeuwen, T. (2012). Single vs. swarm detection of microparticles and exosomes by flow cytometry. J. Thrombosis Haemost. 10, 919-930. doi: 10.1111/j.1538-7836.2012. 04683.x

Van Deun, J., Mestdagh, P., Sormunen, R., Cocquyt, V., Vermaelen, K., Vandesompele, J., et al. (2014). The impact of disparate isolation methods for extracellular vesicles on downstream RNA profiling. J. Extracell. Vesicles. 3:24858. doi: $10.3402 /$ jev.v3.24858

van Niel, G., D'Angelo, G., and Raposo, G. (2018). Shedding light on the cell biology of extracellular vesicles. Nat. Rev. Mol. Cell Biol. 19, 213-228. doi: 10.1038/nrm.2017.125

Vargas Romero, P., Cialfi, S., Palermo, R., De Blasio, C., Checquolo, S., Bellavia, D., et al. (2015). The deregulated expression of miR-125b in acute myeloid leukemia is dependent on the transcription factor C/EBP $\alpha$. Leukemia 29, 2442-2445. doi: 10.1038/leu.2015.117

Villarroya-Beltri, C., Gutiérrez-Vázquez, C., Sánchez-Cabo, F., Pérez-Hernández, D., Vázquez, J., Martin-Cofreces, N., et al. (2013). Sumoylated hnRNPA2B1 controls the sorting of miRNAs into exosomes through binding to specific motifs. Nat. Commun. 4, 1-10.

Viola, S., Traer, E., Huan, J., Hornick, N. I., Tyner, J. W., Agarwal, A., et al. (2016). Alterations in acute myeloid leukaemia bone marrow stromal cell exosome content coincide with gains in tyrosine kinase inhibitor resistance. $\mathrm{Br}$. J. Haematol. 172, 983-986. doi: 10.1111/bjh.13551

Voso, M. T., Ottone, T., Lavorgna, S., Venditti, A., Maurillo, L., Lo-Coco, F., et al. (2019). MRD in AML: the role of new techniques. Front. Oncol. 9:655.

Wang, D., Ming, X., Xu, J., and Xiao, Y. (2021). Circ_0009910 shuttled by exosomes regulates proliferation, cell cycle and apoptosis of acute myeloid leukemia cells by regulating miR-5195-3p/GRB10 axis. Hematol. Oncol. 39, 390-400. doi: $10.1002 /$ hon. 2874 
Wang, M., Zhou, L., Yu, F., Zhang, Y., Li, P., and Wang, K. (2019). The functional roles of exosomal long non-coding RNAs in cancer. Cell. Mol. Life Sci. 76, 2059-2076.

Welsh, J. A., Holloway, J. A., Wilkinson, J. S., and Englyst, N. A. (2017). Extracellular vesicle flow cytometry analysis and standardization. Front. Cell Dev. Biol. 5:78. doi: 10.3389/fcell.2017.00078

Wiese, M., and Daver, N. (2018). Unmet clinical needs and economic burden of disease in the treatment landscape of acute myeloid leukemia. Am. J. Manag. Care 24, S347-S355.

Witwer, K. W., Buzás, E. I., Bemis, L. T., Bora, A., Lässer, C., Lötvall, J., et al. (2013). Standardization of sample collection, isolation and analysis methods in extracellular vesicle research. J. Extracell. Vesicles. 2:20360. doi: 10.3402/jev. v2i0.20360

Wojtuszkiewicz, A., Schuurhuis, G. J., Kessler, F. L., Piersma, S. R., Knol, J. C., Pham, T. V., et al. (2016). Exosomes secreted by apoptosis-resistant Acute Myeloid Leukemia (AML) blasts harbor regulatory network proteins potentially involved in antagonism of apoptosis. Mol. Cell. Proteom. 15, 1281-1298. doi: 10.1074/mcp.m115.052944

Wolf, P. (1967). The nature and significance of platelet products in human plasma. Br. J. Haematol. 13, 269-288. doi: 10.1111/j.1365-2141.1967.tb08741.x

Xiao, M., Li, J., Li, W., Wang, Y., Wu, F., Xi, Y., et al. (2017). MicroRNAs activate gene transcription epigenetically as an enhancer trigger. RNA Biol. 14, 1326-1334. doi: 10.1080/15476286.2015.1112487

Xin, H., Katakowski, M., Wang, F., Qian, J.-Y., Liu, X. S., Ali, M. M., et al. (2017). MicroRNA-17-92 cluster in exosomes enhance neuroplasticity and functional recovery after stroke in rats. Stroke 48, 747-753. doi: 10.1161/strokeaha.116. 015204

Xu, L. H., Guo, Y., Zhang, X. L., Chen, J. J., and Hu, S. Y. (2016). Bloodbased circulating MicroRNAs are potential diagnostic biomarkers for leukemia: result from a meta-analysis. Cell. Physiol. Biochem. 38, 939-949. doi: 10.1159/ 000443046

Xu, R., Rai, A., Chen, M., Suwakulsiri, W., Greening, D. W., and Simpson, R. J. (2018). Extracellular vesicles in cancer - implications for future improvements in cancer care. Nat. Rev. Clin. Oncol. 15, 617-638. doi: 10.1038/s41571-0180036-9

Xu, X., Lai, Y., and Hua, Z.-C. (2019). Apoptosis and apoptotic body: disease message and therapeutic target potentials. Biosci. Rep. 39:BSR20180992.

Xue, H., Hua, L.-M., Guo, M., and Luo, J.-M. (2014). SHIP1 is targeted by miR-155 in acute myeloid leukemia. Oncol. Rep. 32, 2253-2259. doi: 10.3892/or.2014. 3435
Yu, X., Harris, S. L., and Levine, A. J. (2006). The regulation of exosome secretion: a novel function of the p53 protein. Cancer Res. 66, 4795-4801. doi: 10.1158/ 0008-5472.can-05-4579

Yuan, T., Shi, C., Xu, W., Yang, H.-L., Xia, B., and Tian, C. (2021). Extracellular vesicles derived from $\mathrm{T}$-cell acute lymphoblastic leukemia inhibit osteogenic differentiation of bone marrow mesenchymal stem cells via miR-34a-5p. Endocr. J. 2021, EJ21-EJ25.

Zaborowski, M. P., Balaj, L., Breakefield, X. O., and Lai, C. P. (2015). Extracellular vesicles: composition, biological relevance, and methods of study. Bioscience. 65, 783-797. doi: 10.1093/biosci/biv084

Zhang, H., Luo, X. Q., Feng, D. D., Zhang, X. J., Wu, J., Zheng, Y. S., et al. (2011). Upregulation of microRNA-125b contributes to leukemogenesis and increases drug resistance in pediatric acute promyelocytic leukemia. Mol. Cancer 10:108. doi: 10.1186/1476-4598-10-108

Zhao, C., Du, F., Zhao, Y., Wang, S., and Qi, L. (2019). Acute myeloid leukemia cells secrete microRNA-4532-containing exosomes to mediate normal hematopoiesis in hematopoietic stem cells by activating the LDOC1-dependent STAT3 signaling pathway. Stem Cell Res. Ther. 10, 1-12.

Zomer, A., Maynard, C., Verweij, F. J., Kamermans, A., Schäfer, R., Beerling, E., et al. (2015). In vivo imaging reveals extracellular vesicle-mediated phenocopying of metastatic behavior. Cell 161, 1046-1057. doi: 10.1016/j.cell. 2015.04.042

Conflict of Interest: The authors declare that the research was conducted in the absence of any commercial or financial relationships that could be construed as a potential conflict of interest.

Publisher's Note: All claims expressed in this article are solely those of the authors and do not necessarily represent those of their affiliated organizations, or those of the publisher, the editors and the reviewers. Any product that may be evaluated in this article, or claim that may be made by its manufacturer, is not guaranteed or endorsed by the publisher.

Copyright (c) 2021 Izadirad, Huang, Jafari, Hamidieh, Gharehbaghian, Li, Jafari and Chen. This is an open-access article distributed under the terms of the Creative Commons Attribution License (CC BY). The use, distribution or reproduction in other forums is permitted, provided the original author(s) and the copyright owner(s) are credited and that the original publication in this journal is cited, in accordance with accepted academic practice. No use, distribution or reproduction is permitted which does not comply with these terms. 